\title{
Tumor pulmonar acompañado de infarto agudo de miocardio y embolismo arterial en las extremidades inferiores
}

\author{
Jichun Liu ${ }^{\mathrm{a}}$ Hao Chen ${ }^{\mathrm{b}}$ Xiangrong Xie ${ }^{\mathrm{a}} \quad$ Yuwen Yang $^{\mathrm{a}}$ Tang Shengxing ${ }^{\mathrm{a}}$ \\ a Departamento de Cardiología, Primer Hospital Afiliado del Colegio Médico Wannan, Hospital Yijishan del Colegio Médico Wannan, \\ Wuhu, Anhui, República Popular China; ${ }^{\mathrm{b}}$ Departamento de Patologí, Colegio Médico Wannan, Wuhu, Anhui, República Popular China
}

\author{
Palabras clave \\ Infarto agudo de miocardio - Tumor pulmonar . \\ Embolismo arterial $\cdot$ Reporte de caso
}

\section{Resumen}

Antecedentes: La embolización de un tumor pulmonar que conduzca a un infarto agudo de miocardio (IAM) es poco frecuente. Se han reportado casos previos de embolización de un tumor pulmonar en la arteria coronaria. En este trabajo describimos un caso de embolización de tumor pulmonar que causó la ocurrencia simultánea de IAM y embolismo arterial en las extremidades inferiores.

Presentación del caso: Un paciente de 64 años ingresó en el departamento de emergencias refiriendo dolor en el tórax, y se le diagnóstico IAM. Una ecocardiografía mostró una masa en la aurícula izquierda, y se especuló que sería un mixoma. Una angiografía coronaria de emergencia no mostró evidencia de aterosclerosis. Al segundo día de su ingreso, se diagnosticó embolismo arterial en las extremidades inferiores. Inicialmente, especulamos que el mixoma de la aurícula izquierda había causado un embolismo, que condujo al IAM y el embolismo arterial en las extremidades inferiores. Sin embargo, la causa real de ambas condiciones resultó ser un tumor pulmonar. Desgraciadamente, el paciente abandonó el tratamiento cuando supo de su enfermedad y muró tres días después del alta hospitalaria.

Conclusiones: El embolismo de un tumor pulmonar es una causa extremadamente rara de IAM. Aún más raro es el caso aquí presentado, en el que el embolismo de un tumor pulmonar causó IAM y embolismo arterial en las extremidades inferiores. Los profesionales clínicos deben reconocer el embolismo de tumor pulmonar como una causa potencial de IAM.

(c) $2021 \mathrm{El} /$ los autor/es

\section{Antecedentes}

El infarto agudo de miocardio (IAM) usualmente es causado por la ruptura o la erosión de una placa, o por nódulos en las arterias coronarias, aunque existen otras etiologías poco comunes [1]. El tromboembolismo es una complicación común en pacientes con cáncer. Según reportes previos, $4.5 \%$ de los pacientes desarrollan tromboembolismo venoso, y $1.5 \%$ de los pacientes desarrollan tromboembolismo arterial; estos padecimientos pueden ser resultado del estado hipercoagulable asociado con varios cánceres. El embolismo arterial agudo debido a un émbolo tumoral es una complicación poco frecuente en pacientes con cáncer [2], y aún más rara es que la embolización de un tumor cause IAM. Previamente se ha reportado la embolización de un tumor pulmonar en la arteria coronaria $[3,4,5]$. En este trabajo, reportamos el caso de un émbolo en un tumor canceroso de pulmón, que condujo a la aparición simultánea de IAM y un embolismo arterial en las extremidades inferiores.

\section{Presentación del caso}

Un hombre de 64 años, sin hipertensión ni diabetes, ingresó en el departamento de emergencias, manifestando dolor opresivo en el pecho acompañado de sudoración profusa durante al menos cua- 

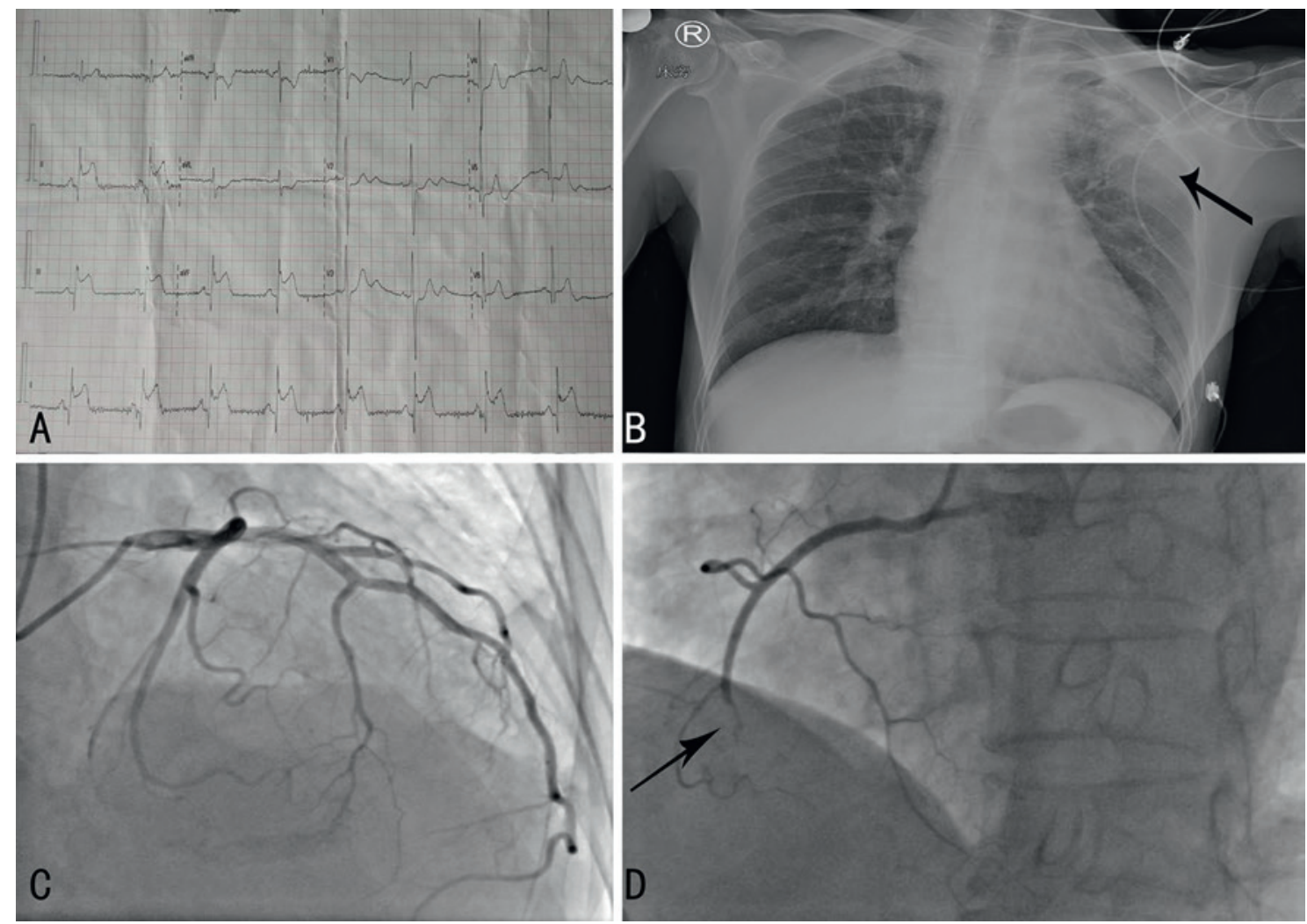

Fig. 1. Resultados del electrocardiograma, radiografía torácica anteroposterior a pie de cama y angiografía coronaria. a El electrocardiograma mostró un ritmo sinusal normal y elevaciones en el segmento ST en las derivaciones II, III y aVF. b Una radiografía torácica anteroposterior mostró lo que se sospechó era neumonía en el pulmón superior izquierdo (flecha negra). c En una angiografía coronaria se encontraron anormalidades en la arteria coronaria izquierda. El segmento medio a distal de la arteria coronaria derecha estaba completamente ocluido (flecha negra).

tro horas tras la admisión. Su temperatura era $36.1^{\circ} \mathrm{C}$; frecuencia respiratoria: 22 respiraciones/minuto; frecuencia cardiaca: 81 latidos/minuto; presión arterial: 114/68 mmHg, y la saturación de oxígeno en el dedo fue $100 \%$ en el aire ambiental. Sus niveles rápidos de troponina I fueron iguales a $4.83 \mathrm{ng} / \mathrm{ml}$.

Un electrocardiograma reveló un ritmo sinusal normal y elevaciones en el segmento ST en las derivaciones II, III y aVF (Fig. 1a). Una ecocardiografía realizada a pie de cama reveló un movimiento anormal en la pared ventricular inferior izquierda y una masa en la aurícula izquierda, que se especuló podría ser un mixoma. Una radiografía torácica posteroanterior realizada a pie de cama mostró lo que se sospechó era neumonía en el pulmón superior izquierdo (Fig. 1b). El paciente recibió un diagnóstico de IAM. Una angiografía coronaria de emergencia no mostró evidencia de aterosclerosis, pero reveló que el segmento medio a distal de la arteria coronaria derecha se encontraba completamente ocluido (Fig. 1d), lo que indicó un embolismo sin manifestación aguda de aterosclerosis.

A pesar de la inyección intracoronaria de tirofibán, aspiración y dilatación con globo, no se recuperó el flujo sanguíneo en el extremo distal de la arteria coronaria derecha, ni se extrajo trombo alguno. Tras la intervención coronaria percutánea, se dio al paciente terapia antiplaquetaria, de regulación de lípidos y anticoagulante.
En se segundo día tras la admisión, el paciente comenzó a sufrir dolor en ambas extremidades inferiores, acompañado de baja temperatura dérmica y hemoptisis. Una ultrasonografía Doppler de las extremidades inferiores practicada al pie da cama mostró una embolización incompleta de la arteria poplítea y la arteria tibial anterior de la extremidad inferior derecha. Una angiografía de emergencia de las arterias en los miembros inferiores reveló que el nacimiento de la arteria femoral profunda y la arteria poplítea se encontraban completamente ocluidos, así como el segmento distal de la arteria tibial posterior izquierda y la arteria fibular (Fig. 2a-c).

Para prevenir la necrosis isquémica de las extremidades inferiores, se realizó una arteriotomía en dichas extremidades bajo anestesia general, y se retiró una gran cantidad de tejido y trombos. Debido a la hemoptisis, se realizó una broncoscopía con fibra óptica bajo anestesia general, y los resultados mostraron la presencia de un extenso coágulo de sangre en el bronquio izquierdo y las vías respiratorias subsecuentes. Para determinar la dirección de crecimiento de la masa en la aurícula izquierda, también se practicó una ecocardiografía transesofágica, la cual mostró que la masa en la aurícula izquierda creció desde la entrada de la vena pulmonar superior (Fig. 3d).

El examen patológico subsecuente del tejido y el trombo empleando la tinción H\&E reveló necrosis por coagulación con células con 

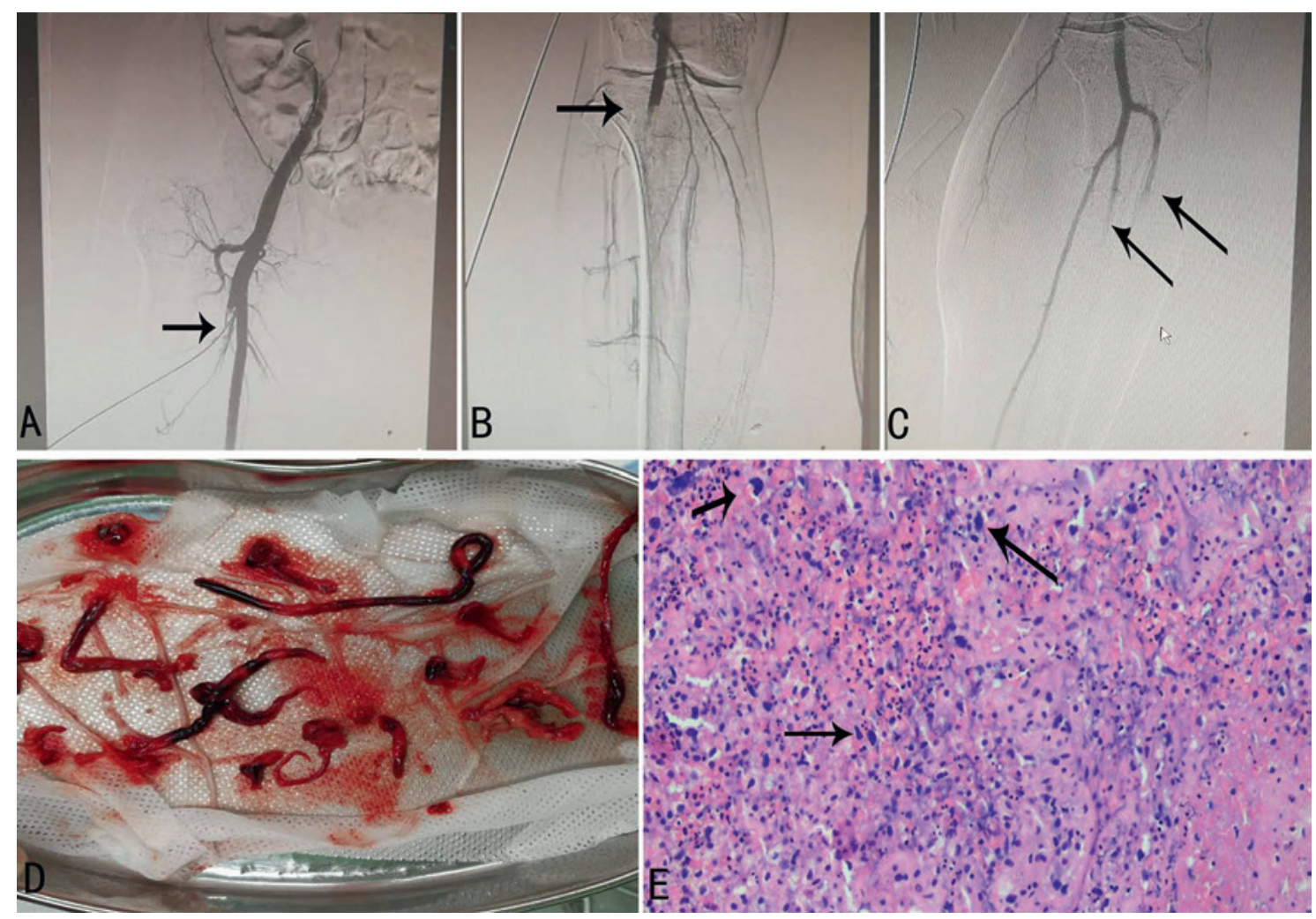

Fig. 2. Resultados de la angiografía de las arterias de las extremidades inferiores y tejido de las extremidades inferiores, así como examen patológico del tejido y el trombo. a El nacimiento de la arteria femoral profunda derecha se encontraba ocluido (flecha negra). b Oclusión de la arteria poplítea derecha (flecha negra). c El segmento distal de la arteria tibial posterior izquierda y la arteria fibular se encontraba completamente ocluido (flecha negra). d Tejido de la extremidad inferior y trombo. e Necrosis por coagulación con células con atipia (tinción H\&, 200 ×, flecha negra).

Fig. 3. Exploración por tomografía computarizada (TC) del tórax y ecocardiografía transesofágica. a Lesión sólida con bordes irregulares en el lóbulo superior izquierdo del pulmón (flecha blanca). b La vena pulmonar superior izquierda se encontraba ocluida por material sólido (flecha blanca). c Masa sólida en la aurícula izquierda (flecha blanca). d Una ecocardiografía transesofágica reveló una masa sólida en la aurícula izquierda, creciendo desde la entrada de la vena pulmonar superior izquierda (flecha blanca).

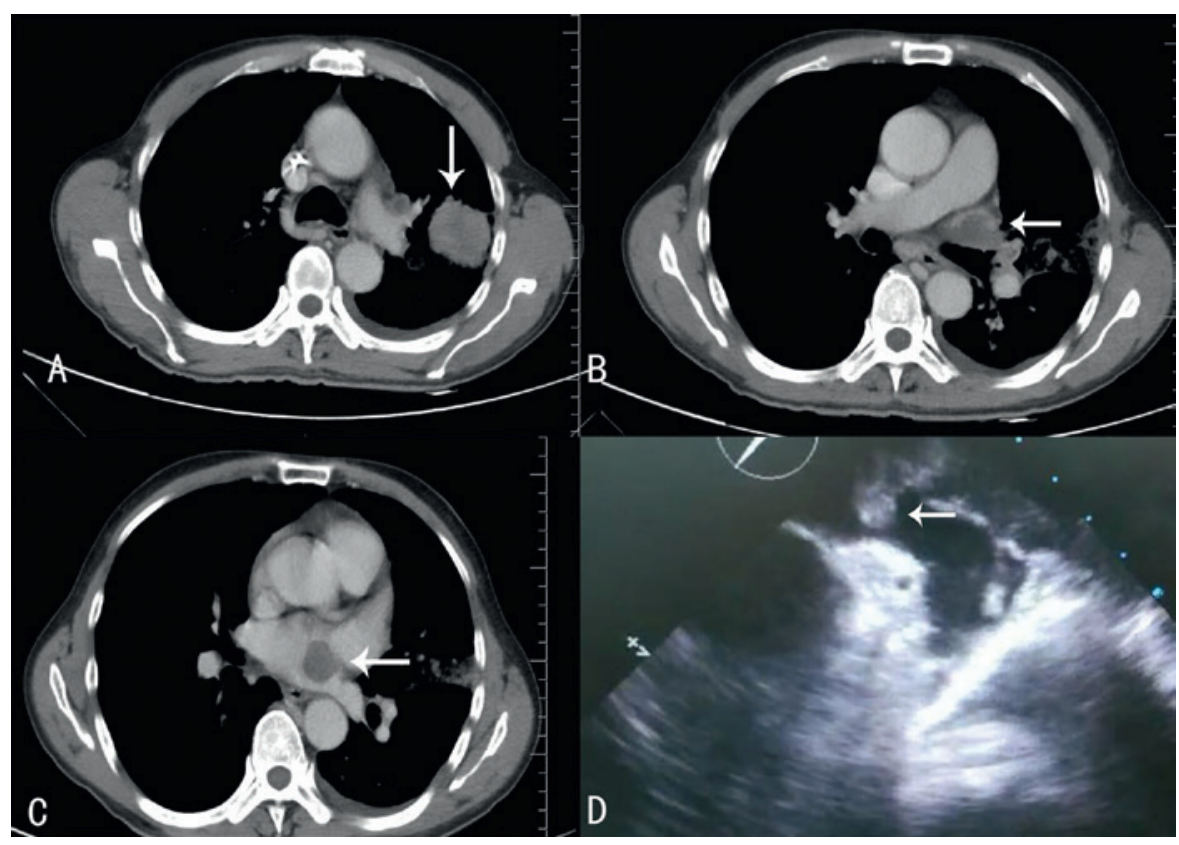

atipia (Fig. 2e). La tinción inmunohistoquímica fue positiva para CD163 (Fig. 4a) y negativa para SMA, CD31, ERG, CK7, TTF, Napsin-A, CK5/6, P63 y P40. El índice de proliferación (Ki-67) fue aproximadamente igual a $2 \%$ (Fig. $4 \mathrm{~b}$ ).
Una exploración por tomografía computarizada (TC) del tórax reveló una lesión sólida en el lóbulo superior del pulmón izquierdo, con bordes irregulares, que se especuló sería un tumor sólido (etapa IVA). El tumor parecía ingresar en la aurícula izquierda 
Fig. 4. Histología del tejido y el trombo. a El tejido y el trombo mostraron resultados positivos de inmunohistoquímica para CD163 (200 ×). b El índice de proliferación (Ki-67) fue aproximadamente igual a 2\% (inmunotinción, $200 \times$ ).
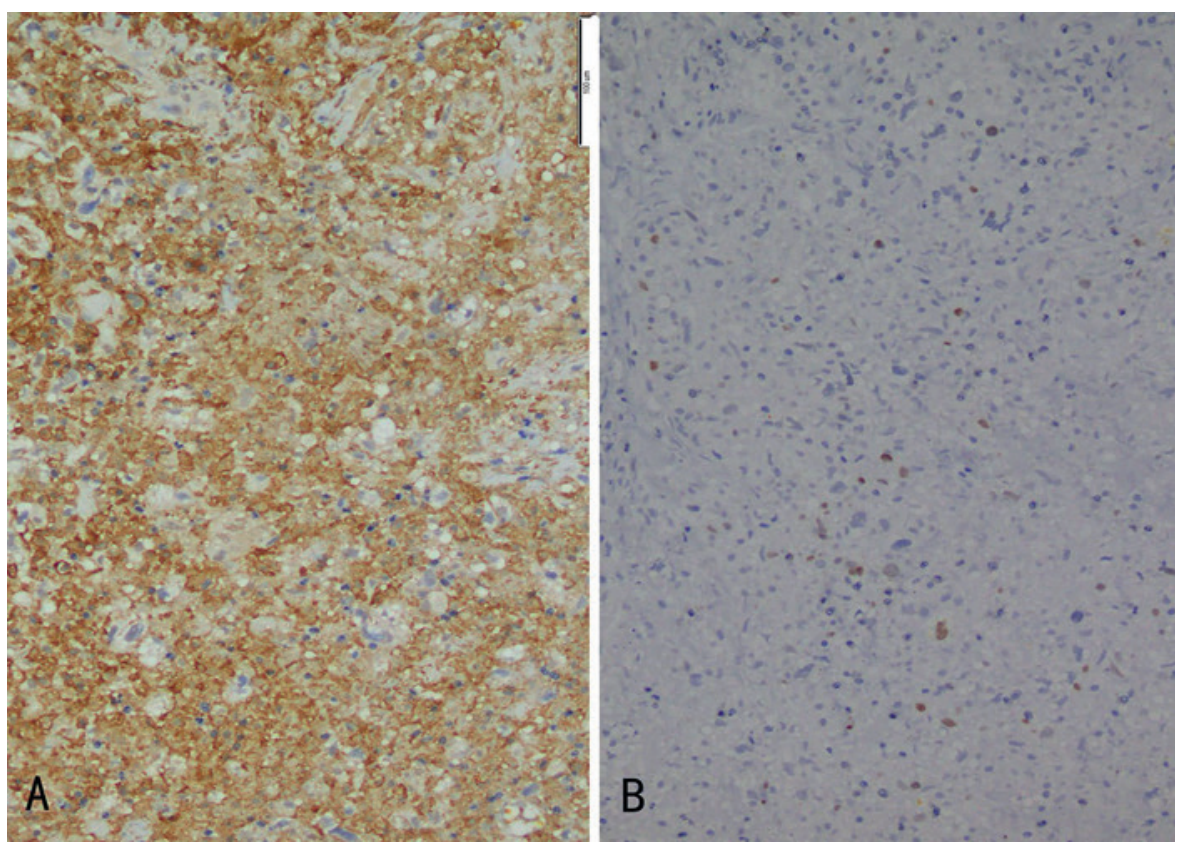

desde la vena pulmonar superior izquierda, que aparecía completamente ocluida debido a la presencia de material sólido en su luz (Fig. 3a-c). Con base en el historial médico del paciente y el examen, se sospechó la presencia de un embolismo tumoral. Desgraciadamente, cuando el paciente supo de su enfermedad, abandonó el tratamiento y salió del hospital. Una semana después, se contactó por teléfono a la familia del paciente, quienes informaron que éste había muerto tres días después del alta hospitalaria.

\section{Discusión y conclusiones}

El embolismo arterial coronario (EC), en el que material obstructivo ingresa en la arteria coronaria, bloquea el flujo sanguíneo y causa isquemia, es una causa poco común de IAM. La prevalencia de IAM a consecuencia de EC es $2.9 \%$. El IAM resultante de EC puede ser causado por mixoma en la aurícula izquierda, fibrilación auricular, cardiomiopatía, valvulopatía, neoplasias, endocarditis infecciosa y defectos en el septo auricular. De acuerdo con los datos disponibles, no existe diferencia significativa en la incidencia de EC entre la rama anterior descendente izquierda, la rama circunfleja izquierda y la arteria coronaria derecha [6].

Las fuentes más comunes de émbolos arteriales de origen tumoral son los tumores primarios de pulmón [7]. Generalmente, los tumores pulmonares se extienden al corazón por diseminación hematógena, a través del sistema linfático, por invasión directa y por difusión intracavitaria por la vena cava inferior o las venas pulmonares. Los tumores de pulmón se extienden al corazón por el sistema linfático con mayor frecuencia que a través de las venas pulmonares. La entrada de tumores pulmonares a la aurícula izquierda por la vena pulmonar es poco común [8]. El embolismo arterial agudo causado por un émbolo tumoral es una complicación rara en pacientes con cáncer. El mecanismo aceptado del embolismo arterial en tumores de pulmón malignos involucra la ex- pulsión de los nidos tumorales fuera del sistema luego de invadir las venas pulmonares [2]. Se han reportado casos de IAM causado por émbolos derivados de tumores pulmonares $[5,9]$. La embolización de un tumor pulmonar también puede ocurrir en otros sistemas arteriales, entre ellos la aorta, la arteria femoral, arterias en las extremidades y la arteria mesentérica [7]. En este caso, se realizó la aspiración del trombo en la arteria coronaria, aunque no pudo removerse el trombo. Suponemos que la causa fue un pequeño orificio en la pared del catéter de succión, que fue incapaz de extraer émbolos tumorales extensos.

El embolismo tumoral es una causa extremadamente rara de IAM. Aún más raro es el caso aquí reportado, en el que un embolismo tumoral produjo IAM y embolismo arterial en las extremidades inferiores. Sin embargo, el profesional clínico debe reconocer que un embolismo tumoral es una causa potencial de IAM.

\section{Conflicto de interés}

Los autores declaran que no tienen intereses en competencia.

\section{Información sobre licencias}

Jichun Liu, Hao Chen, Xiangrong Xie, Yuwen Yang, Shengxing Tang: Tumor pulmonar acompañado de infarto agudo de miocardio y embolismo arterial en las extremidades inferiores. BMC Cardiovasc Disord. 2020; 20(1):482. (https://doi/10.1186/s12872-020-01770-0). ( ${ }^{\circ} \mathrm{El} / \mathrm{los}$ autor/es 2020 (traducción; abreviaturas, agradecimientos, contribuciones de los autores, financiamiento, disponibilidad de datos y materiales, aprobación ética y consentimiento del paciente, permiso para su publicación, nota del editor abreviada), protegido por la licencia CC BY 4.0 (https://creativecommons.org/licenses/by/4.0/deed.es).

\section{Referencias}

Las referencias están disponibles en www.karger.com/Article/Fulltext/ 000513672 . 